\title{
Preventive Medicine: A "Cure" for the Healthcare Crisis
}

\author{
TRANSCRIBED AND ADAPTED FOR PUBLICATION IN DISEASE MANAGEMENT \\ BY JANICE L. CLARKE, R.N. AND DEBORAH C. MEIRIS, MEDICAL WRITERS, \\ DEPARTMENT OF HEALTH POLICY, JEFFERSON MEDICAL COLLEGE, \\ PHILADELPHIA, PENNSYLVANIA
}

This supplement is based on the proceedings of the U.S. Preventive Medicine, Inc. (USPM) Advisory Board meeting, which took place on March 17, 2006 in Philadelphia, Pennsylvania. This supplement was supported by an educational grant from USPM. 


\section{EDITORIAL}

\section{DAVid B. NASH, MD, MBA}

Few would dispute the premise that prevention, early detection, and early intervention form the first line of defense on the disease management (DM) continuum. That being the case, our national statistics on preventive health should be raising concerns throughout the industry. The US healthcare delivery system continues to fall woefully short of its prevention targets. On the international scene, the United States lags behind countries with less wealth and less technological savvy. Commentaries abound on the problems, but recently I became aware of an organization with an exciting goal and a novel solution for bringing preventive medicine into the mainstream.

U.S. Preventive Medicine, Inc. (USPM) was founded by Christopher Fey, a former president and CEO of HealthCare USA, a multistate health maintenance organization, and senior officer of Coventry Health Care Corporation. A number of years ago, Mr. Fey had a life-altering experience. He witnessed his brother-in-law, a 39-year-old man in seemingly excellent physical condition, suffer a massive stroke that resulted in permanent right-sided paralysis, and speech and memory impairment. Following the event, physicians concluded that his brother-in-law's risk factors could have been identified and his disease state detected by means of available technological screening devices. His was a condition for which effective drug therapy and other interventions were available. This event and its consequences were preventable.

Having experienced firsthand the devastating consequences of a "broken" system that fails to respond until a condition produces symptoms, Mr. Fey became an "evangelist" for prevention and early detection. In founding USPM, he translated an interesting concept into an innovative model for preventive health in a consumer-driven market.

In the following pages we provide a brief history of and current status report on the state of preventive health in the United States, and we present an overview of this company's solution as one example of the untapped potential for innovation in the delivery of preventive services. I hope that the information contained herein will inspire you and your colleagues to join the conversation about the direction the United States will take with regard to improving access to screening and preventive services and enriching the lives of all citizens. As always, I welcome your comments. I can be reached at David.Nash@jefferson.edu.

\section{PREVENTIVE HEALTH IN THE UNITED STATES}

A look back

Over the past few decades, the politics of prevention have undergone an evolution. In the late 1960s and early 1970s, prevention was at the forefront of healthcare reform. With very little controversy, vaccination, promotion of lifestyle changes, mass screenings, and safety regulations became widely accepted as strategies for improving health and reducing expenditures. By the mid-1980s, some unanticipated outcomes of these strategies had become apparent. Serious and permanent injuries were attributed to the vaccines used for immunizations. Lifestyle factors 
were being used as the basis for raising health insurance rates and/or denying consumer eligibility for disability benefits. Preventive screenings had become suspect as potential tools for a range of activities, from denying employment to selective abortion. Lawsuits were brought charging that occupational safety standards were being used to exclude people from certain jobs. ${ }^{1}$

In the late 1980s, the US Preventive Services Task Force (USPSTF) launched the first edition of its Guide to Clinical Preventive Services under the auspices of the Agency for Healthcare Research and Quality (AHRQ). ${ }^{2}$ The guide contained recommendations that helped to establish the importance of including prevention in primary health care, ensuring health plan coverage for effective preventive services, and holding healthcare providers and systems accountable for delivering preventive care. The guide has been updated periodically, and the guidelines contained therein continue to form the basis of clinical standards for professional societies, healthcare organizations, and medical quality review groups. Current USPSTF recommendations for preventive services are listed in Table 1.

With the growth of managed care organizations in the 1980s and 1990s, insurance coverage for preventive services was introduced, and a new emphasis was placed on disease prevention and health education. Primary care providers were encouraged, and often "rewarded," for focusing on screening and prevention. In the early 1990s, disease state management programs began to appear, providing aggressive prevention of disease progression and complications as well as treatment for chronic conditions.

Today the United States is the world's richest, most technologically advanced nation, but US preventive health statistics continue to fall dramatically short of expectations. The associated costs are alarmingly high. Consider the following:

- More than 20 million Americans (7\% of the population) have diabetes, though experts estimate that nearly $30 \%$ of this population remains undiagnosed. ${ }^{3}$

- According to the National Kidney Foundation, more than 20 million Americans have undetected moderate chronic kidney disease and another 20 million are at risk. $^{4}$

- According to the American Heart Association, the lifetime risk of developing hypertension is approximately $90 \%$ for adults between the ages of 55 and 65; one third of those affected are not aware they have this condition. ${ }^{5}$

- Chronic obstructive pulmonary disease (COPD) also warrants attention because large numbers of cases are undiagnosed. In 2001, at a time when more than 12 million adults were diagnosed with COPD, experts estimate that an additional 16 million remained undiagnosed and therefore untreated. Failure to treat can be fatal-COPD is the fourth leading cause of death in the United States-and it's costly; in 2000, national COPD costs totaled more than $\$ 32$ billion, split nearly evenly between direct and indirect costs. ${ }^{6}$

- At least half of the deaths from cancers (564,830 projected in 2006) could be prevented by greater use of established screening tests and the application of existing knowledge. ${ }^{7}$

In their recent study, "Disease and Disadvantage in the United States and in En- 
TABLE 1

U.S. Preventive Services Task Force (USPSTF) Recommended Preventive Services (2005)

\begin{tabular}{|c|c|c|c|c|}
\hline \multirow[b]{2}{*}{ Recommendations } & \multicolumn{2}{|l|}{ Adults } & \multicolumn{2}{|c|}{ Special populations } \\
\hline & Men & Women & $\begin{array}{l}\text { Pregnant } \\
\text { women }\end{array}$ & Children \\
\hline Alcohol misuse screening and behavioral counseling interventions & $\mathrm{X}$ & $\mathrm{X}$ & $X$ & \\
\hline Aspirin for the primary prevention of cardiovascular events ${ }^{a}$ & $X$ & $X$ & & \\
\hline Bacteriuria, screening for asymptomatic & & & $X$ & \\
\hline Breast cancer, chemoprevention ${ }^{b}$ & & $\mathrm{X}$ & & \\
\hline Breast cancer, screening ${ }^{c}$ & & $X$ & & \\
\hline Breastfeeding, behavioral interventions to promote $^{\mathrm{d}}$ & & $X$ & $X$ & \\
\hline Cervical cancer, screeninge & & $X$ & & \\
\hline Chlamydial infection, screening ${ }^{\mathrm{f}, \mathrm{g}}$ & & $X$ & $X$ & \\
\hline Colorectal cancer, screeningh & $\mathrm{X}$ & $X$ & & \\
\hline Dental caries in preschool children, prevention ${ }^{\mathrm{i}}$ & & & & $\mathrm{X}$ \\
\hline Depression, screening $\mathrm{j}$ & $\mathrm{X}$ & $X$ & & \\
\hline Diabetes mellitus in adults, screening for type $2^{\mathrm{k}}$ & $X$ & $X$ & & \\
\hline $\begin{array}{l}\text { Diet, behavioral counseling in primary care to promote a } \\
\text { healthy }\end{array}$ & $X$ & $X$ & & \\
\hline Hepatitis B virus infection, screening ${ }^{\mathrm{m}}$ & & & $\mathrm{X}$ & \\
\hline High blood pressure screening & $\mathrm{X}$ & $\mathrm{X}$ & & \\
\hline Lipid disorders, screening ${ }^{\text {n,o }}$ & $X$ & $X$ & & \\
\hline Obesity in adults, screeningp & $X$ & $X$ & & \\
\hline Osteoporosis in postmenopausal women, screening ${ }^{\mathrm{q}}$ & & $X$ & & \\
\hline Rh (D) incompatibility, screening ${ }^{\mathrm{r}, \mathrm{s}}$ & & & $\mathrm{X}$ & \\
\hline Syphilis infection, screeningt & $\mathrm{X}$ & $\mathrm{X}$ & $X$ & \\
\hline Tobacco use and tobacco-caused disease, counseling to prevent ${ }^{\mathrm{u}, \mathrm{v}}$ & $X$ & $x$ & $x$ & \\
\hline $\begin{array}{l}\text { Visual impairment in children younger than age } 5 \text { years, } \\
\text { screening }{ }^{w}\end{array}$ & & & & $X$ \\
\hline
\end{tabular}

adults at increased risk for coronary heart disease.

${ }^{b}$ Discuss with women at high risk for breast cancer and at low risk for adverse effects of chemoprevention.

'Mammography every 1-2 years for women 40 and older.

dStructured education and behavioral counseling programs.

eWomen who have been sexually active and have a cervix.

fSexually active women 25 and younger and other asymptomatic women at increased risk for infection.

gAsymptomatic pregnant women 25 and younger and others at increased risk.

hMen and women 50 and older.

iPrescribe oral fluoride supplementation at currently recommended doses to preschool children older than 6 months whose primary water source is deficient in fluoride.

In clinical practices with systems to assure accurate diagnoses, effective treatment, and follow-up.

${ }^{k}$ Adults with hypertension or hyperlipidemia.

${ }^{1}$ Adults with hyperlipidemia and other known risk factors for cardiovascular and diet-related chronic disease.

mPregnant women at first prenatal visit.

nMen 35 and older, and women 45 and older.

'Younger adults with other risk factors for coronary disease; screening for lipid disorders to include measurement of total cholesterol and high-density lipoprotein cholesterol.

PIntensive counseling and behavioral interventions to promote sustained weight loss for obese adults.

qWomen 65 and older, and women 60 and older at increased risk for osteoporotic fractures.

${ }^{\mathrm{r} B l o o d}$ typing and antibody testing at first pregnancy-related visit.

sRepeated antibody testing for unsensitized Rh (D)-negative women at 24-28 weeks gestation unless biological father is known to be Rh (D) negative.

tPersons at increased risk and all pregnant women.

uTobacco cessation interventions for those who use tobacco.

vAugmented pregnancy-tailored counseling to pregnant women who smoke.

wTo detect amblyopia, strabismus, and defects in visual acuity.

From Guide to Clinical Preventive Services (2005). Available at: www.ahrq.gov/clinic/pocketgd/gcps1.htm. 
gland," Banks and colleagues concluded that the US population in late middle age is less healthy than the equivalent British population, with respect to self-reported chronic conditions and biological markers of disease, despite considerably greater per capita spending on health care (US \$5274 vs. UK $\$ 2164$ [adjusted]. $)^{8}$ The differences reportedly exist at all levels of socioeconomic status.

As a nation, our failure to detect conditions and initiate early intervention has resulted in a precipitous rise in the prevalence and severity of chronic diseases. ${ }^{9}$ Chronic conditions account for $70 \%$ of all deaths in the United States, and the costs associated with chronic conditions account for more than $60 \%$ of the nation's medical care costs. Perhaps most unsettling is that, for a majority of chronic diseases, onset can be delayed or progression limited by avoiding risky behaviors, increasing physical activity, and obtaining life-saving screening services.

Why is preventive health care so far from where it should be in the world's wealthiest nation? There are a variety of possible explanations. Some commercial insurance carriers ration coverage by adhering to conservative standards and recommendations. In the public sector, some restrictions on coverage for preventive services are even more stringent. For instance, Medicare does not cover preventive services for senior citizens, and most Medicaid carriers restrict preventive care to minimal standards and recommendations. But the problem is more pervasive. A recent survey of 153,000 adults concluded that only $3 \%$ of US citizens adhere to the four key healthy lifestyle characteristics (ie, not smoking, maintaining healthy weight, eating adequate amounts of fruits and vegetables, and exercising regularly). ${ }^{10}$ Almost $10 \%$ of respondents admitted that they did not adhere to any of the four characteristics.

Consumer-driven healthcare and its effect on preventive health

Several market forces are beginning to align, creating ideal conditions for a major transition in healthcare delivery:

1. The projected impact of the aging "babyboom" population

2. The ever-escalating cost of providing health care

3. The increasing role of consumers in managing all aspects of their health

Today more than 80 million "babyboomers" are beginning to attain senior status with a previously unheard-of passion for enjoying healthy, active lives. The impact of this generation on established markets, business, and society promises to be challenging. On the positive side, medical and information technology are advancing at a rapid pace, enabling ever higher degrees of personalized care for a population demanding proactive care to ensure longevity and quality of life. On the flip side, the cost of care continues to outpace the economy's ability to pay for "traditional" care. In the final analysis, this population may leverage their considerable wealth and political influence to make the necessary changes in the system.

In "Wealth Patterns among Elderly Americans: Implications for Healthcare Affordability," Knickman and colleagues point out that, at nearly $17 \%$ of the gross domestic product, health care is the largest single industry in the United States. ${ }^{11}$ If this trend continues, an aging population could 
overwhelm the economy's ability to support health care. The authors reason that a traditional approach (ie, "sick care") delivers care when treatment options are most costly and outcomes are most limited. They observe that business and consumer markets are beginning to challenge the assumption that medical treatment should be reserved for the "sick." Some examples cited are:

- Employers are beginning to understand the value of "buying health" in reducing absenteeism and "presenteeism." They are becoming interested in investing in a healthy, productive workforce.

- Consumers want to extend their physically and mentally active years, maintaining or improving their quality of life as they age.

- Government must act to lower the longterm cost of care.

Proactive preventive care is increasingly viewed as both a logical and a necessary alternative. Early diagnostic tests, assessments, and aggressive intervention in advance of symptoms provide an appealing alternative, given the lower cost of these types of interventions and the high potential for positive outcomes at the presymptomatic stage.

As consumers assume greater financial responsibility for the cost of their personal health care, they are likely to demand greater influence over how their health dollars are spent. Consumers may demand optional tests and procedures in order to establish, in concert with their physicians, an accurate baseline for their health. Such a baseline can be useful when making purchase decisions on the type and level of health coverage needed. Baseline and ongoing trend information will offer consumers' clinical teams a more effective means of monitoring their patients' health and changes in conditions.

The trend toward consumer-driven health care is beginning to reset expectations regarding who pays for prevention. Although some employers continue to provide coverage that includes preventive services, in most cases consumers are the purchasers of preventive care. The challenge for consumers is identifying how and where to purchase prevention.

\section{Can prevention fit into the traditional care model?}

One solution is for consumers to go to a preventive care center for reliable, accurate, and comprehensive individualized testing, and then go to a physician for interpretation of those test results and development of a medical management plan. The critical factors for the success of such a solution are a uniform, consistent, and accessible experience for the consumer and for the physician.

Market forces are already driving these changes. A combination of factors (ie, the financial transfer to consumers and the trend toward proactive care) are shifting the focus and responsibility for individual health to the consumer. The new healthcare consumer is assuming a larger role in his or her personal health. For example, Heath Savings Accounts (HSAs), in which health spending is at the discretion of the individual, are on the rise.

In recent years, there has been an unprecedented rise in consumer involvement in healthcare purchasing and decision making. The information necessary for making informed healthcare decisions is becoming readily available via the Internet, personal health record (PHR) technology, public service materials, and from healthcare insurers and employers. 


\section{ONE COMPANY'S INNOVATIVE SOLUTION: THE U.S. PREVENTIVE MEDICINE, INC. MODEL}

Although navigating the healthcare system in today's environment is often challenging and frustrating, consumers have had no alternative but to "take charge" in the not-so-user-friendly world of health care. U.S. Preventive Medicine, Inc. (USPM) sees its role as offering consumers the services of a "captain" to help them plot their course for healthier, active lives-with an emphasis on prevention.

USPM was founded on the premise that healthcare service delivery is entering a period of transition in the United States. Traditionally, the focus of care has been reactive, with services delivered only when a patient's illness becomes symptomatic. As the system evolves, the focus has been shifting increasingly to proactive, preventive care in which consumers are treated on a presymptomatic basis-a concept that dovetails with the philosophy of disease management.

\section{Culture of prevention}

The US healthcare system is grounded in a medical infrastructure wherein care is provided and reimbursed based on a perceived doctor-patient relationship in which "prevention" is defined simply: "eat right, exercise, take vitamins, and see the doctor regularly." USPM's goal is to reorganize the prevention arena by driving a transition from "legacy of treatment" to a "culture of prevention." Moving to a culture of prevention entails expanding the definition to include understanding what is going on inside the body (eg, genetics, blood chemistries).

\section{Building a prevention ecosystem}

The USPM model takes a comprehensive approach to prevention, employing multiple components and a multipart mission to create a "prevention ecosystem." The broad goals include:

- Creating a national system of consumer- and employer-focused preventive care centers established in conjunction with hospitals and physician groups. The centers for preventive medicine (CPM) will provide consumers and employers with point-of-care preventive services for health diagnostics, assessment, counseling, and medical intervention as appropriate.

- Educating, informing, and motivating consumers and employers to pursue prevention lifestyles and to utilize virtual and physical preventive services. In a television program format with a focus on "entertainment," the company seeks to influence consumer health behaviors in areas such as fitness, healthy aging, and wellness. Theoretically, the convenience of receiving this information and education in the home environment will increase the likelihood of consumer involvement.

- Functioning as a holding company for units in the "prevention ecosystem." Units will include a licensing group for CPMs; The Prevention Channel; online prevention services (ie, Preventionchannel.com); group prevention services (ie, disease management services); and research (eg, data mining, clinical research). USPM Holding Company revenue would flow from licensing fees, 
profit and revenue sharing, advertising, products and services, "permissionbased" data mining, clinical research, disease management, and value appreciation.

USPM sees its role as a catalyst in pulling divergent interests (ie, employers, consumers, government) together in a business model focused on creating a culture of prevention. Mr. Fey admits, "It will not be a quick or cheap fix, but it needs to happen. We need a model in which every player wins." An important element in making this transition is recognizing the differences between individual consumer health and population health; a model for individual health must go beyond a population model approach (eg, educating the population to exercise and take vitamins). An individual health model must incorporate technologies that screen for and monitor chronic conditions. "Cardiovascular disease takes 30 years to kill a person. A person with this condition needs to know about it as early as possible and seek appropriate treatment to achieve the best outcome."

Promoting the cause of individualized prevention is the first step. The real dilemma lies in moving the healthcare delivery system toward providing all necessary preventive services. A recent article on women's health reported an alarmingly high rate of missed diagnoses of heart disease in women, but there is no public outcry. Preventive health rarely hits the radar screen. "People don't walk around asking for the nearest—or best—preventive health center."

The Prevention Channel. USPM's model envisions a communication component with a novel intervention-a cable channel named "The Prevention Channel." The Prevention Channel is designed to deliver prevention messages in an entertaining way, on the order of the History Channel or the Discovery Channel. Programs or "soap operas" will focus on common health elements that a viewer can change by taking some action. For example, diabetes may be woven into a story in such a way that viewers begin to understand this health issue on a deeper, personal level. By identifying with the characters, viewers may become better equipped to recognize possible early symptoms of common chronic illness in themselves and in their family members. Prevention topics will include heart disease, stroke, cancer screening, and aging gracefully. The message- "70 is the new middle age."

PreventionChannel.com. The Prevention Channel programs may motivate consumers to take some action regarding their personal health. Viewers with concerns about their health will be directed to virtual prevention services online (ie, PreventionChannel.com) where they may complete user-friendly, comprehensive assessments that predict risk levels for various conditions. Consumers, in collaboration with their healthcare providers, can use this knowledge to determine appropriate interventions and measure results.

Online and paper programs have been designed to improve participant health and postpone morbidity by reducing health risks, promoting self-efficacy, and instilling self-care skills. There are basic programs and programs designed for the needs of special populations. These interventions are grounded in the science of behavioral change and draw upon the expertise of practicing clinicians and health educators. 
A medical director oversees panels of clinicians who continually review medical and scientific literature for the most current information and incorporate it into the organization's processes.

\section{USPM CPMS}

The core of the USPM system is a national network of CPMs. With support from a national advisory board, a medical advisory board, and relationships with national medical organizations (eg, ACPM), these centers are designed to bridge the gap between traditional, reactive medicine and modern, proactive medicine. CPMs have the potential for positive impact on the quantity and quality of preventive health in the communities they serve by "defining the preventive experience, providing a physical presence, and creating credibility, content, and authority."

In the traditional, illness-focused model, the healthcare or hospital system is designed to intervene at late, acute, or symptomatic stages when outcomes are poor and costs are high. The CPM model focuses on preventing disease from the outset by identifying it at the earliest point when outcomes are best and costs are lowest. USPM partners with hospitals and physician groups in co-ownership arrangements of its branded CPMs. Conceptually, the model represents a change in process rather than a "frontal assault." The company contracts with thousands of physicians and invites the participation of all who meet USPM's professional and clinical standards. The goal is for the CPMs to become dominant regional providers of consumer- and employer-focused prevention, wellness, and healthy aging services.

The three components of the CPM core structure are USPM, the hospital partner, and the physician partners (Fig. 1). Ideally, the CPM is located in a multispecialty building that is near or appended to, but not in, the hospital. Fifteen hundred square feet is adequate space, and a CPM is designed to use staff, imaging, laboratory, and cardiology services that are already in place at the hospital.

CPM services are rendered by physicians in a clinical environment where assessment and prevention protocols are tailored to individual patients rather than populations. The challenge is to create a one-stop/onesource for preventive care and the goal is to establish 150 CPMs across the United States over a five-year period that will serve approximately 1000 patients per year at each center. At full capacity, this represents approximately $0.1 \%$ of the US population. The CPM does not replace the patient's relationship with his or her doctor. Rather, it facilitates and enhances the opportunity for early detection, and encourages diligence among those with increased risk factors.

The company envisions a geographical distribution for CPMs that encompasses both urban and rural areas. One hundred fifty markets have been identified with population sizes as low as 150,000. Currently, a CPM is operating in Sioux City, Iowa. In terms of staffing, the hospital added only one full-time employee, an RN administrator. Patient data is stored on a secure drive. There is a private lounge and changing rooms, television, and computers with Internet access. A concierge escorts the patient from one diagnostic area to another. Flexibility in scheduling assures little downtime between tests. Lifestyle coaching by an RN is included for one year following diagnostic testing.

Preventive service packages are available 
THE CENTER FOR PREVENTIVE MEDICINE ${ }^{\circledR}$

BUSINESS OVERVIEW

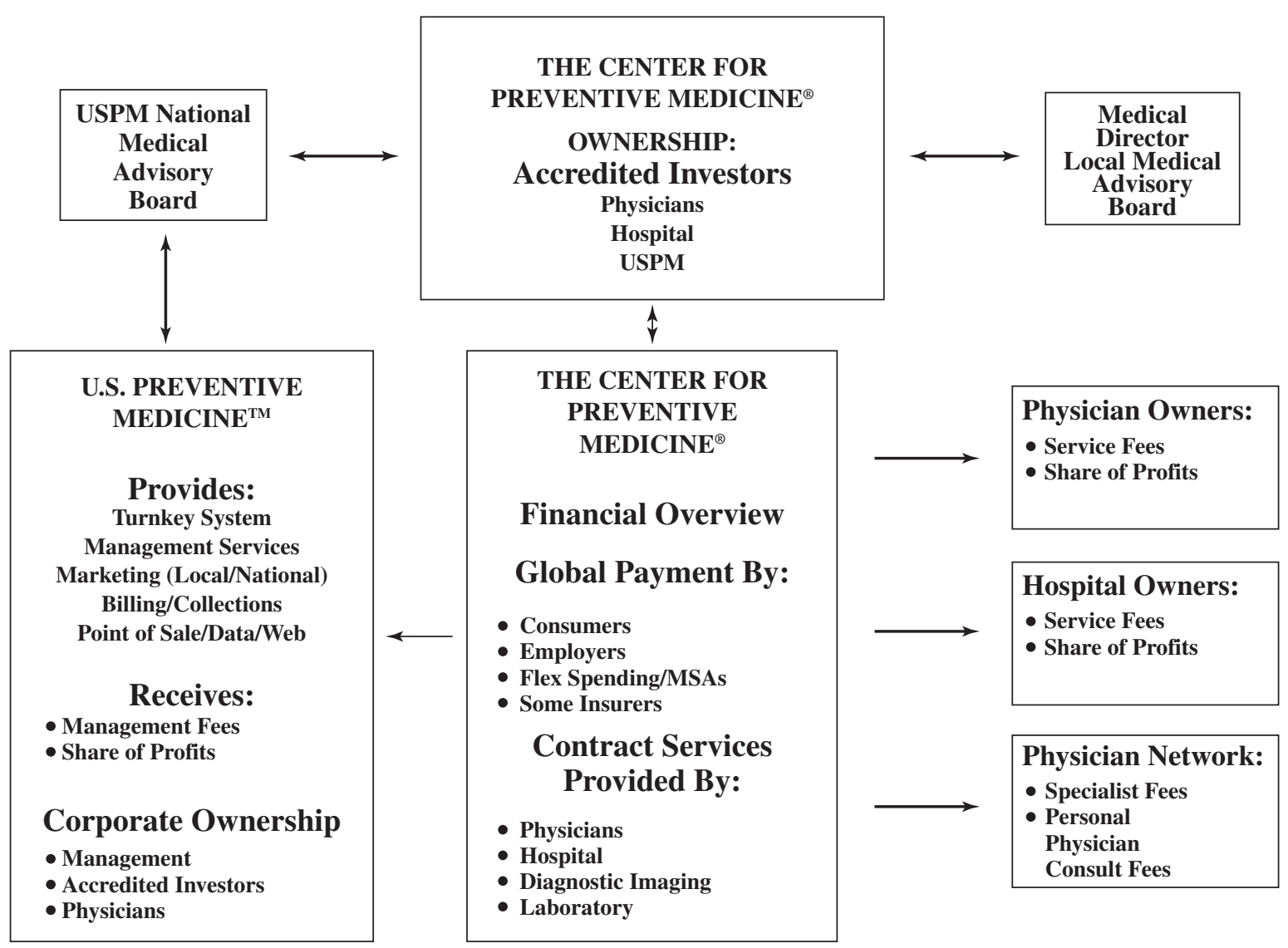

FIG. 1. U.S. Preventive Medicine, Inc. (USPM) center for preventive medicine (CPM) structure.

at different price points across the spectrum depending on the number and types of screenings and/or tests performed. At the high end, the cost per patient at a CPM ranges from $\$ 1425$ to $\$ 5950$, with cardiology-specific packages ranging from $\$ 825$ to $\$ 3415$. For those desiring lower-end preventive services, employers may purchase packages for as little as $\$ 10$ per employee per year for basic risk stratification.

In practical terms, the US healthcare system is comprised of contracts and services. The CPM model seeks to extend contracts and services for preventive care beyond their current bounds, leading to an appre- ciable increase in wellness and continuity of care.

\section{National Advisory Board Reaction to the USPM model}

With a variety of stakeholders involved in this enterprise, it is important to recognize different perspectives and address issues exclusive to each stakeholder. USPM sought the expertise of the Department of Health Policy at Jefferson Medical College in convening a National Advisory Board to serve as impartial consults to the company's strategy team (Table 2). The goal 
TABLE 2

U.S. Preventive Medicine, Inc. (USPM) Advisory Board

F. Kenneth Ackerman, Jr., F.A.C.H.E., F.A.C.M.P.E.

President

Clark Counsulting Healthcare Group

Minneapolis, Minnesota

David Auerbach, M.D., M.B.A.

Associate Professor, Internal Medicine-

General Medicine

University of Texas, Southwestern

Medical School

Dallas, Texas

Robert N. Butler, M.D.

President and Chief Executive Officer International Longevity Center-USA

New York, New York

Anthony DiMarino, M.D.

Division Chief

Gastroenterology and Hepatology

Thomas Jefferson University Hospital

Philadelphia, Pennsylvania

James Fries, M.D.

Professor of Medicine (Emeritus)

Stanford University

Stanford, California

Robert Goldberg, Ph.D.

Director

Center for Medical Progress

Manhattan Institute

New York, New York

Lynn Helmer, M.D., F.A.C.P., M.B.A.,

F.A.C.P.E.

President

DRD Consulting, Inc.

Haddon Heights, New Jersey
Maulik Joshi, Dr.P.H.

Former President and Chief Executive Officer

Delmarva Foundation for Medical Care, Inc.

Easton, Maryland

Matias Klein

President and Chief Technology Officer

Ethidium Health Systems

Huntingdon Valley, Pennsylvania

Ronald Loeppke, M.D., M.P.H.

Chief Medical Officer and Executive

Vice President

CorSolutions, Inc.

Brentwood, Tennessee

S. Jay Olshansky, Ph.D.

Professor

School of Public Health

The Population Research Center at NORC and

The University of Chicago

Chicago, Illinois

Jason Pride, C.E.A.

Vice President and Director of Research

The Haverford Trust Company

Radnor, Pennsylvania

Ann Scott Blouin, Ph.D., R.N.

Principal

Deloitte Consulting LLP

Chicago, Illinois

Richard Tewksbury

Manager Business Development

TRION Group

King of Prussia, Pennsylvania 
was to obtain objective feedback on the company's broad "ecosystem" approach to transforming preventive health care, and to elicit critique regarding the CPMs from clinical, financial, and business perspectives.

The National Advisory Board, comprised of national-caliber healthcare industry leaders (up to 12 members representing medical specialties, healthcare administration, and employers), met in Philadelphia, Pennsylvania on March 17, 2006. At its initial meeting, the board reviewed USPM concepts and the existing business model, identified potential risks and barriers, discussed the application of evidence-based practices, and outlined an outcomes research strategy. The board is expected to reconvene periodically to provide guidance during the development of the CPMs.

The chief questions posed were: Can this model work? Does it make economic and clinical sense? Board members agreed that the USPM model represents a very innovative view of preventive medicine. They noted that the concepts represent a departure from the conventional "public health" approach to prevention, focusing instead on privatization of the preventive health model. One member observed, "The model is an interesting interface between high-tech and high-touch."

The USPM National Advisory Board noted that the CPM product is highly individualized for the client/consumer but must be rigorously standardized across centers and regions (eg, a test for detecting melanoma cannot be available at one center and not another). If the screening tool (eg, a health status and risk assessment) recommends a screening test or study to consumers, it must be integrated into the preventive options offered.

The opportunity exists to redefine US preventive medicine in terms of what it can be.
The World Health Organization defines health in broad terms, balancing physical, emotional, social, and spiritual elements. The Board suggested that a comprehensive definition of preventive medicine be built around this model. Such a definition would span the continuum from primary prevention to early intervention to tertiary prevention (ie, treating to prevent complications).

The Board encouraged the company to continue to focus on helping individuals to manage their own health and to negotiate the healthcare process. Several members advised that the preventive medicine program include such things as advance directives and end-of-life care in addition to the risk assessments and screening tests. They questioned "What is done with a positive finding?" and, "Where will consumers be told to 'start taking a baby aspirin'?" With this model, these fall into the healthcare provider's domain. "We want an organization that is both accessible and economicalone that avoids duplicating effort." The company is working on standardizing the information reported on each test performed, and the company's Web site will provide additional detail for physicians and for individuals who have concerns.

Employer-related issues. The chief issue for employers is the differential in the value propositions. "Productivity is the new currency for employers looking to 'buy health' for their workforces." Employers are seeking products that cover all health care for all of their employees-both prevention and treatment. The concierge-style CPMs are impressive, but they are designed and priced for executives. Board members encouraged the company to focus similar attention on continued development of products and services employers might purchase for their general workforces. 
Physician-related issues. Several Board members noted potential challenges at the provider level in the model. "What are the implications if a patient is diagnosed with a genetic disorder or predisposition?" The Board also commented on the potential for resistance from physicians when subclinical or presumptive diagnostics are introduced. "Physicians resist having their patients get tests outside of the doctor-patient context."

The Board discussed the importance of educating physicians regarding the significance of specific tests and counseling scores. The key message was that maintaining good relationships with physicians will be critical to the success of the model. "Always ask the name of the person's doctor and report to that doctor before the patient does."

Ethical issues. As they are presently conceived, CPMs are accessible to and affordable by "high-end" consumers (ie, employer-sponsored executives and consumers willing and able to pay $\$ 1500$ out of pocket). Although other components are more universally accessible (eg, The Prevention Channel), the Board cautioned that the CPMs may give the appearance of elitism or "cherry picking." They encouraged the company to focus some of its innovative capital on a solution to address prevention for the huge uninsured US population, as well as those insured by Medicare and Medicaid. Additional opportunities include fashioning programs for specific employers (eg, police, firefighters) and programs that consider regional culture, making certain that customer service training extends to the physician.

While sensitive to this critique, USPM reported that experience at its prototype CPM in Sioux City, Iowa suggests that educated consumers are interested in invest- ing in personal prevention and early detection. Also, plans are under way to provide affordable screening programs via a Web-based health status and risk assessment tool, and education via "The Prevention Channel."

Partnering. The Board advised USPM to choose its hospital and physician partners very carefully to ensure the best clinical quality in a given region. "Selection of partners will set the tone relative to perceived quality."

Business model. The model assumes unused capacity at hospitals. For example, the hospital with the fully operational CPM has an entire wing dedicated to prevention and fitness. Board members commented that many hospitals in the United States and Canada have no excess capacity.

\section{Medical content and standards}

The Board emphasized the importance of establishing and maintaining standards of care and evaluating quality across CPMs. They raised questions about the practice of individual medicine as contrasted with population medicine. Even though most people are risk averse there are exceptions, and the permissible degree of variation from the standard must be established (eg, a consumer who desires a liver biopsy every three months when the risk of dying from the test is greater than the risk of dying from the disease).

The Board stressed the value of involving clinical partners in clinical content determinations and package designs. There should be a valid reason for doing each test-one that is understood by the consumer and the physician. Additional demands on physician time may be alleviated in part by developing decision trees. 
Additional "prevention package" offerings suggested by the Board were a cardiology service package, and a women's preventive health package (ie, reproductive health, cardiovascular, osteoporosis screening).

Evidence-based medicine. The healthcare industry is seeking evidence-based medicine. Purchasers, providers, and consumers expect it. Wherever possible, there should be evidence to support the program. Increasingly, the onus of responsibility for an individual's health is on the consumer. Because of this, it is important to educate and help manage the expectations of consumers.

The Board noted that the model deals as much with "early discovery" as with prevention. In deciding whether to be totally evidence based (ie, adhere to relatively conservative USPSTF recommendations) or to follow suggestions from the most current literature (ie, practice cutting-edge prevention and early detection), the Board advised taking a proactive approach. "Framingham cannot predict for individuals. It is only useful for populations."

Outcomes measures and studies. Outcomes measurements are essential for demonstrating the positive effects of the model-for example, a reduction in morbidity or mortality that results in increased productivity. The Board strongly recommended that USPM create a research arm to track participants in programs and analyze data for changes in behavior and health risks. They noted rich sources of data including existing data from CPMs, online health status surveys, and risk assessments. In addition to studies, the Board stressed the importance of conducting scholarly reviews of the literature.

Observational studies might be designed to evaluate the effect of USPM's programs and answer important questions such as:
- If the expected outcome is extended "healthy life," gauge and monitor this.

- Does participation reduce the risk of death?

- Does participation increase the quality of life?

- Does living a healthier life lead to greater accumulation of wealth?

- Does living a healthier life result in fewer sick days and more productivity at work?

- What is an acceptable outcome? Does early detection always result in a better outcome?

Preventive care is a long-term commitment. One would expect differences in costs for a person aged 50 versus a person aged 60 . A study comparing out-of-pocket and other costs for participants versus nonparticipants at various ages would be of great interest.

An information technology system is an integral part of a research solution. Health status assessments, procedures, and test results form a core data set and an umbrella questionnaire could be administered annually to all program participants with evaluation embedded into the system. Another element to consider is a PHR on the Web site that would enable integration of the individual participant's prevention experience and test results.

By the meeting's end, the key points were synthesized in the form of a proposed mission statement:

1. Improve American health.

2. Improve individual health via lifestyle changes.

3. Detect disease at an early stage.

4. Evaluate outcomes of prevention efforts thoroughly.

5. Conduct scholarly reviews of the literature.

6. Ensure adequate financial return for preventive services. 


\section{CONCLUSION}

The US healthcare system's failure to meet targets for prevention and early detection of chronic conditions has raised concerns throughout the industry. Although improvements have resulted from the efforts of health plans, disease management companies, and public sector agencies, preventive health care continues to fall short of expectations for a variety of reasons including system issues (eg, rationing or restricting coverage for preventive services by insurers) and consumer behavior issues (eg, failure to adhere to key healthy lifestyle characteristics). In this supplement, we have described one innovative strategy for improving the amount and quality of prevention, early detection, and healthy lifestyle education - the creation of a national network of CPMs delivering consistent, reliable preventive services and solutions to consumers across the country. We also present a national expert panel's reaction to the strategy. The USPM model is of particular interest because of its broad, encompassing scope-the "ecosystem" concept-and its novel approaches to physician and hospital partnering, diagnostic/screening test packaging and delivery, and consumer engagement and education regarding preventive health.

In the months since the National Advisory Board met to evaluate and critique its model, USPM executives have worked to address the board's recommendations (eg, seeking, as partners, institutions with a "passion for prevention," taking steps toward building a research arm).

Although we have highlighted one approach in this supplement, there are other possible routes to improving preventive health nationwide. The overarching message is that refocusing on prevention and early detection may be the first stage in a "cure" for the healthcare crisis in the United States.

\section{REFERENCES}

1. Stone DA. The resistible rise of preventive medicine. J Health Polit Policy Law 1986;11:671-696.

2. U.S. Preventive Services Task Force. Highlights: U.S. Preventive Services Task Force Guide. Available at: http://ahrq.gov/clinic/uspstf.htm. Accessed July 6, 2006.

3. National Institute of Diabetes and Digestive and Kidney Diseases (NIDDK). National diabetes statistics. Available at: http://diabetes.niddk. nih.gov/dm/pubs/statistics/index.htm. Accessed September 10, 2006.

4. U.S. Renal Data System. USRDS 2005 annual data report. Available at: www.usrds.org/adr.htm. Accessed September 10, 2006.

5. American Heart Association. Heart disease and stroke statistics-2003 update. Dallas, TX: American Heart Association, 2003.
6. National Institutes of Health. Data fact sheet: chronic obstructive pulmonary disease-2003. $\mathrm{NIH}$ publication no. 03-5229. Bethesda, MD: NIH.

7. American Cancer Society. Cancer prevention and early detection facts and figures, 2006. Available at: www.cancer.org/docroot/STT/ content/STT_1x_Cancer_Prevention_and_Earl y_Detection_Facts_Figures_2006.asp. Accessed August 23, 2006.

8. Banks J, Marmot M, Oldfield Z, Smith JP. Disease and disadvantage in the United States and in England. JAMA 2006;295:2037-2045.

9. National Center for Chronic Disease Prevention and Health Promotion. Chronic disease overview. Available at: www.cdc.gov/nccdphp/ overview.htm. Accessed July 11, 2006. 
10. Reeves MJ, Rafferty AP. Healthy lifestyle characteristics among adults in the United States, 2000. Arch Intern Med 2005;165:854-857.

11. Knickman JR, Hunt KA, Snell EK, Alecxih LM, Kennell DL. Wealth patterns among elderly Americans: implications for health care affordability. Health Aff (Millwood) 2003; 22:168-174.
Address correspondence to: Janice L. Clarke Department of Health Policy Jefferson Medical College 1015 Walnut St., Ste 115 Philadelphia, PA 19107 E-mail: janice.clarke@jefferson.edu 